\title{
S25. A Study on Disease showed Singular Cerebroangiographical Findings seemed to be due to New Collateral Circulation
}

\author{
Jiro Suzuki, Masayoshi Kowada, Masayoshi AsahI, \\ Akira TAKaKU and Norio HARADA \\ 2nd Surgical Clinic, Tohoku University
}

We have recently experienced 6 young cases which had many netform vessels in brainstem cerebroangographically with stenosis or occlusion of the carotid siphon. These onset symptom were severe migraine, choreatic movements, temporary hemiplegia, seizure attack, subarachnoid haemorrhage or intracerebral bleeding. We researched these causes, studying past and familial histories, rheumatic reactions, hormonal examinations etc. And we reported the histological findings of one autopsy case.

\section{S26. On Operative Treatment of Hypertensive Intracerebral Hemorrhage}

\author{
Takao Mitsuno, Haruyuki Kanaya, Seiya Sirakata, Yasuhiro KuzU, \\ Kanji Saotome, Seiji Shimmaru, Ken-ichi Ohsawa, Yasumasa \\ Ishikawa, Hideo Yamasaki, Setsuo Ono, Humio Nomura, \\ Hideki OnOdera, Masamichi Jin, Sadao KobaYashi, \\ Iwao SAIKI and Yutaka HoRIE \\ 2nd Dept. of Surgery, Iwate Medical College
}

Operative treatment of hypertensive intracerebral hemorrhage has been performed since 1903 by Cushing, but the operative mortality was so high that the procedure made little progress.

The authors operated upon 26 cases of such patients. Total evacuation of hematoma was done in 17 cases, partial removal in 7 cases, and ventricular tapping only in 2 cases. Total evacuation with trephination of $4.1 \mathrm{~cm}$ diameter is the operation of choice. Although all of the patients were the most severe cases in coma, including 24 cases of secondary intraventricular hemorrhage, 14 cases recovered.

From these results and autopsy findings of dead cases after operation, the effectiveness of the surgical treatment were discussed. 